\title{
Cross-migrative commutative aggregation functions with respect to Archimedean triangular norms
}

\author{
Wenwen Zong ${ }^{a}$, Yong Su${ }^{b}$, Hua-Wen Liu ${ }^{c}$ \\ ${ }^{a}$ School of Mathematical Sciences, University of Jinan, Jinan, Shandong 250022, China, zongwen198811@163.com \\ ${ }^{b}$ School of Science, Jiangnan University, Wuxi, Jiangsu 214122, China, yongsu88@163.com \\ ${ }^{c}$ School of Mathematics, Shandong University, Jinan, Shandong 250100, China, hw.liu@sdu.edu.cn
}

\begin{abstract}
Functional equations involving aggregation functions play an important role in fuzzy sets and fuzzy logic theory. The cross-migrative equation is a weaker form of the classical commuting equation. This paper is devoted to exploring cross-migrative commutative aggregation functions with respect to Archimedean triangular norms.
\end{abstract}

Keywords: Aggregation functions, Functional equation, Triangular norms, Crossmigrativity

\section{Introduction}

Functional equations involving aggregation functions play an important role in fuzzy set theory, fuzzy logic, the theories of non-additive integrals, and aggregation process. For example, the (conditional) distributivity equation naturally appears in several types of constructions of integrals (see $\S 5.6$ in [2] for details); the bisymmetry equation expresses the condition that aggregation of all elements of any square matrix can be performed first on the rows, then on the columns or conversely (see pp. 40 in [2]); the commuting equation is important in any two-stepped information aggregation procedure [7]. In this paper, we focus on the cross-migrativity equation, which is a weaker form of the commuting equation.

Fodor et al. [1] introduced the cross-migrativity equation, as weaker form of the commuting equation, and presented the complete characterization of the class of continuous triangular norms, which are crossmigrative with respect to some strict or nilpotent triangular norms, respectively. Later on, the sufficient and necessary conditions that a t-subnorm with a continuous additive generator is $\alpha$-cross-migrative with respect to $T_{M}, T_{P}$ and $T_{L}$, respectively, have been presented by Wang and Qin [8]. In this paper, we focus on the general cases, that is to say, an aggregation function being $\alpha$-cross-migrative with respect to an Archimedean t-norm.

\section{Preliminaries}

For more information regarding triangular norms, see the monograph [5]. Otherwise, the following section addresses several necessary notions.

Aggregation functions were proposed to aggregate several values into just one. However, we restrict ourselves to the two-dimensional case of aggregation operations. A binary aggregation function is a mapping, $A:[0,1]^{2} \rightarrow[0,1]$, which is increasing with respect to both variables, and fulfills the boundary conditions of $A(0,0)=0$ and $A(1,1)=1$.

A triangular norm (t-norm for short) $T$ is a binary operation on $[0,1]$ such that $([0,1], T)$ is an Abelian ordered semigroup with neutral element 1 . Standard examples of t-norms are the minimum $T_{\mathbf{M}}$, the product $T_{\mathbf{P}}$, the Eukasiewicz t-norm $T_{\mathrm{E}}$ given by $T_{\mathrm{E}}(x, y)=$ $\max (x+y-1,0)$, and the drastic product $T_{\mathbf{D}}$ with $T_{\mathbf{D}}(1, x)=T_{\mathbf{D}}(x, 1)=x$, and $T_{\mathbf{D}}(x, y)=0$ otherwise.

A continuous t-norm $T$ is called to be Archimedean if $T(x, x)<x$ for any $x \in(0,1)$. The continuous Archimedean t-norms $T$ are completely characterized by their additive generators [6], i.e., by continuous, strictly decreasing functions $t:[0,1] \rightarrow[0, \infty]$ with $t(1)=0$, via

$$
T(x, y)=t^{(-1)}(t(x)+t(y))
$$

where $t^{(-1)}:[0, \infty] \rightarrow[0,1]$ defined by

$$
t^{(-1)}(z)=t^{-1}(\min (z, t(0)))
$$

is the pseudo-inverse of $t$. A t-norm $T$ is strict if it is continuous and strictly increasing, i.e., $T(x, y)<$ $T(x, z)$ whenever $x>0$ and $y<z$, and is nilpotent if it is continuous and if each $x \in(0,1)$ is a nilpotent 
element. Each additive generator $t$ of a nilpotent tnorm satisfies $t(0)<\infty$, whereas in the strict case we have $t(0)=\infty$.

\section{Main results}

Definition 1 Let $\alpha \in(0,1)$ and $T$ be a t-norm. $A$ commutative aggregation function $A$ is $\alpha$-crossmigrative with respect to $T$ (shortly, $(\alpha, T)$-crossmigrative) if for any $x, y \in[0,1]$ we have

$$
A(T(x, \alpha), y)=T(x, A(\alpha, y)) .
$$

Similar to Lemma 2.2 in [1], the following result follows directly from the commutativity of the considered functions.

Proposition 1 Let $T$ be a t-norm and $A$ be a commutative aggregation function on $[0,1]$. Then we have $T$ is $(\alpha, A)$-cross-migrative if and only if $A$ is $(\alpha, T)$ cross-migrative.

Recall that for each aggregation function $A$, and for each strictly increasing bijection, $\varphi:[0,1] \rightarrow[0,1]$, the function $A_{\varphi}:[0,1]^{2} \rightarrow[0,1]$ defined by

$$
A_{\varphi}(x, y)=\varphi^{-1}(A(\varphi(x), \varphi(y)))
$$

is also an aggregation function. Algebraically speaking, $A$ and $A_{\varphi}$ are isomorphic. Direct checking verifies the following result:

Proposition 2 Let $\varphi:[0,1] \rightarrow[0,1]$ be a strictly increasing bijection, $\alpha \in(0,1), T$ and $A$ be, respectively, a t-norm and an aggregation function on $[0,1]$. Then $A$ is $(\alpha, T)$-cross-migrative if and only if $A_{\varphi}$ is $\left(\varphi^{-1}(\alpha), T_{\varphi}\right)$-cross-migrative.

Taking into account that each strict t-norm is isomorphic to $T_{P}$, and each nilpotent t-norm is isomorphic to $T_{\mathrm{E}}$. Proposition 2 allows us, among others, to extend each result about cross-migrativity with respect to $T_{P}$ and $T_{\mathrm{E}}$ to a result about cross-migrativity with respect to strict and nilpotent t-norms, respectively. Fodor et al. [1] have presented the sufficient and necessary conditions that a continuous t-norm is $\alpha$-crossmigrative with respect to $T_{M}, T_{P}$ and $T_{\mathrm{E}}$, respectively. Later on, the sufficient and necessary conditions that a t-subnorm with a continuous additive generator is $\alpha$-cross-migrative with respect to $T_{M}, T_{P}$ and $T_{\mathrm{E}}$, respectively, have been presented by Wang and Qin [8]. In what follows, we focus on the sufficient and necessary conditions that an aggregation function $A$ is $\alpha$-cross-migrative with respect to $T_{P}$ and $T_{\mathrm{E}}$, respectively.

\subsection{Cross-migrativity with respect to $T_{P}$}

For $\alpha \in(0,1)$ and a commutative aggregation function $A, A$ being $\left(\alpha, T_{P}\right)$-cross-migrative means that for any $(x, y) \in[0,1]^{2}$

$$
A(\alpha x, y)=x A(\alpha, y) .
$$

Obviously, whether or not (1) holds ultimately depends on the values of $A$ on $[0,1]^{2} \backslash[\alpha, 1]^{2}$ but not on $[\alpha, 1]^{2}$.

Proposition 3 Let $\alpha \in(0,1)$ and $A$ be a commutative aggregation function on $[0,1]$. Then $A$ is $\left(\alpha, T_{P}\right)$-cross-migrative if and only if for any $(x, y) \in$ $[0,1]^{2} \backslash[\alpha, 1]^{2}$,

$$
A(x, y)=\frac{f(x) f(y)}{c}
$$

where $c=A(\alpha, \alpha) / \alpha^{2}, f:[0,1] \rightarrow[0, \infty)$ is an increasing function and $f(x)=c x$ for any $x \in[0, \alpha]$.

Proof. The sufficiency is guaranteed from a routine calculation.

Conversely, suppose that $A$ is $\left(\alpha, T_{P}\right)$-cross-migrative. By (1), we necessarily obtain $A(\alpha x, \alpha y)=x y A(\alpha, \alpha)$, i.e., for any $u, v \in[0, \alpha]$,

$$
A(u, v)=c u v .
$$

where $c=\frac{A(\alpha, \alpha)}{\alpha^{2}}$. Consider the function $f:[0,1] \rightarrow$ $[0, \infty)$ defined by

$$
f(x)=\frac{A(\alpha, x)}{\alpha}
$$

for any $x \in[0,1]$. Equation (2) implies that $f$ is an increasing function and $f(x)=c x$ for any $x \in[0, \alpha]$. From (1), we necessarily have for any $x, y \in[0,1]$,

$$
A(\alpha x, y)=\alpha x f(y)
$$

i.e., for any $u \in[0, \alpha]$ and $v \in[0,1]$, we obtain

$$
A(u, v)=\frac{f(u) f(v)}{c} .
$$

By the commutativity of $A$, we have for any $(x, y) \in$ $[0,1]^{2} \backslash[\alpha, 1]^{2}$

$$
A(x, y)=\frac{f(x) f(y)}{c}
$$

Remark 1 Proposition 3 presents the sufficient and necessary conditions that $A$ is $\left(\alpha, T_{P}\right)$-cross-migrative. These conditions are not, however, sufficient to ensure that $A$ is a commutative aggregation function because the values of $A$ on $[\alpha, 1]^{2}$ are unknown. In particular, if $f$ is a strictly increasing bijection, then a stric$t$ t-norm $A(x, y)=f^{-1}(f(x) f(y))$ is $\left(\alpha, T_{P}\right)$-crossmigrative. 
Example 1 Let $\alpha \in(0,1), 0<c<\frac{1}{\alpha}, f(x)=c x$ for any $x \in[0,1]$ and

$$
\begin{aligned}
A(x, y) & = \begin{cases}1 & \text { if } x, y \in[\alpha, 1], \\
\frac{f(x) f(y)}{c} & \text { otherwise }\end{cases} \\
& = \begin{cases}1 & \text { if } x, y \in[\alpha, 1], \\
c x y & \text { otherwise }\end{cases}
\end{aligned}
$$

Then the commutative aggregation function $A$ is $\left(\alpha, T_{P}\right)$-cross-migrative by Proposition 3.

\subsection{Cross-migrativity with respect to $T_{\mathbf{E}}$}

For $\alpha \in(0,1)$ and a commutative aggregation function $A, A$ being $\left(\alpha, T_{\mathrm{E}}\right)$-cross-migrative means that for any $(x, y) \in[0,1]^{2}$

$A(\max (\alpha+x-1,0), y)=\max (x+A(\alpha, y)-1,0)$.

Obviously, (3) only determines the values of $A$ on $[0,1]^{2} \backslash[\alpha, 1]^{2}$ and it is nothing to do with the values of $A$ on $[\alpha, 1]^{2}$.

Proposition 4 Let $\alpha \in(0,1)$ and $A$ be a commutative aggregation function on $[0,1]$. Then $A$ is $\left(\alpha, T_{E}\right)$-cross-migrative if and only if for any $(x, y) \in$ $[0,1]^{2} \backslash[\alpha, 1]^{2}$,

$$
A(x, y)=\max (\min (x, y)+f(\max (x, y))-\alpha, 0),
$$

where $f:[0,1] \rightarrow[0,1]$ is an increasing function satisfying $f(y)=\max (y-\alpha+A(\alpha, \alpha), 0)$ for any $y \in[0, \alpha]$.

Proof. The sufficiency is guaranteed from a routine calculation.

Conversely, suppose that $A$ is $\left(\alpha, T_{\mathrm{E}}\right)$-cross-migrative. By (3), we necessarily have

$$
\begin{aligned}
& A(\max (\alpha+x-1,0), \max (\alpha+y-1,0)) \\
= & \max (x+y-2+A(\alpha, \alpha), 0),
\end{aligned}
$$

i.e., for any $u, v \in[0, \alpha]$

$$
A(u, v)=\max (u+v-2 \alpha+A(\alpha, \alpha), 0) .
$$

Consider the function $f:[0,1] \rightarrow[0,1]$ defined by $f(x)=A(\alpha, x)$ for any $x \in[0,1]$. Then $f$ is an increasing function and $f(x)=\max (x-\alpha+A(\alpha, \alpha), 0)$ for any $x \in[0, \alpha]$ by (4). From (3), we necessarily have for any $x, y \in[0,1]$,

$$
A(\max (\alpha+x-1,0), y)=\max (x+f(y)-1,0)
$$

i.e., for any $u \in[0, \alpha]$ and $v \in[0,1]$, we obtain

$$
A(u, v)=\max (u+f(v)-\alpha, 0) .
$$

The commutativity implies that for any $(x, y) \in$ $[0,1]^{2} \backslash[\alpha, 1]^{2}$,

$$
A(x, y)=\max (\min (x, y)+f(\max (x, y))-\alpha, 0) .
$$

Corollary 1 Let $\alpha \in(0,1), A(\alpha, \alpha)=\alpha$ and $A(\alpha, y) \leq \min (\alpha, y)$ for any $y \in[0,1]$. Then a commutative aggregation function $A$ is $\left(\alpha, T_{E}\right)$-crossmigrative if and only if there exists a commutative aggregation function $A^{\prime}$ such that

$$
A(x, y)= \begin{cases}\alpha T_{\Perp}\left(\frac{x}{\alpha}, \frac{y}{\alpha}\right) & \text { if } x, y \in[0, \alpha], \\ \alpha+(1-\alpha) A^{\prime}\left(\frac{x-\alpha}{1-\alpha}, \frac{y-\alpha}{1-\alpha}\right) & \text { if } x, y \in[\alpha, 1], \\ \min (x, y) & \text { otherwise. }\end{cases}
$$

Proof. The sufficiency is trivial.

Conversely, suppose that $A$ is $\left(\alpha, T_{\mathrm{E}}\right)$-cross-migrative. Consider the function $f:[0,1] \rightarrow[0,1]$ defined by $f(x)=A(\alpha, x)$ for any $x \in[0,1]$. From Proposition 4 , $A(\alpha, \alpha)=\alpha$ and $A(\alpha, y) \leq \min (\alpha, y)$ for any $y \in[0,1]$, we have $f(x)=\min (x, \alpha)$ for any $x \in[0,1]$ and hence, for any $x, y \in[0, \alpha]$ we get

$$
A(x, y)=\max (x+y-\alpha, 0),
$$

and for any $x, y \in[0,1]$ such that $\min (x, y) \leq \alpha \leq$ $\max (x, y)$ we obtain $A(x, y)=\min (x, y)$. Let

$A^{\prime}(x, y)=\frac{A(\alpha+(1-\alpha) x, \alpha+(1-\alpha) y)-\alpha}{1-\alpha} x, y \in[0,1]$.

It is easy to verify that $A^{\prime}$ is a commutative aggregation function. Therefore, $A$ can be presented as (5).

Example 2 Let $\alpha \in(0,1)$.

(i) Consider $A^{\prime}$ as the smallest aggregation function $A_{\perp}$ with $A_{\perp}(1,1)=1$ and $A_{\perp}(x, y)=0$ otherwise. Then Corollary 1 indicates that, $A_{1}$, defined by,

$A_{1}(x, y)= \begin{cases}\max (x+y-\alpha, 0) & \text { if }(x, y) \in[0, \alpha]^{2}, \\ \alpha & \text { if }(x, y) \in[\alpha, 1]^{2} \backslash\{(1,1)\}, \\ \min (x, y) & \text { otherwise, }\end{cases}$

is $\left(\alpha, T_{E}\right)$-cross-migrative.

(ii) If $A^{\prime}=T_{P}$, then Corollary 1 indicates that, $A_{2}$, defined by,

$A_{2}(x, y)= \begin{cases}\max (x+y-\alpha, 0) & \text { if }(x, y) \in[0, \alpha]^{2}, \\ \frac{x y-\alpha y-\alpha x+\alpha}{1-\alpha} & \text { if }(x, y) \in[\alpha, 1]^{2}, \\ \min (x, y) & \text { otherwise, }\end{cases}$

is $\left(\alpha, T_{E}\right)$-cross-migrative.

\section{Acknowledgement}

Support from the National Natural Science Foundation of China (Grant No.11801220), the Natural Science Foundation of Jiangsu Province (Grant No. BK20180590), the Natural Science Foundation of Shandong Province (Grant No.ZR2019BA005), the Fund of Doctoral Program Research of University of Jinan (Grant No. XBS1832) and the Fundamental Research Funds for the Central Universities (Grant No. JUSRP11832) is fully acknowledged. 


\section{References}

[1] J. Fodor, E. P. Klement, R. Mesiar, Crossmigrative triangular norms, International Journal of Intelligent Systems 27 (2012) 411-428.

[2] M. Grabisch, J. L. Marichal, R. Mesiar, E. Pap, Aggregation Functions, Encycl. Math. Appl., vol.127, Cambridge University Press, New York, 2009 .

[3] S. Li, F. Qin, J. Fodor, On the cross-migrativity with respect to continuous t-norms, International Journal of Intelligent Systems, 30 (2015) 550-562.

[4] C. Alsina, M.J. Frank, B. Schweizer, Associative Functions: Triangular Norms and Copulas, World Scientific Publishing Co., Singapore, 2006.

[5] E. P. Klement, R. Mesiar, E. Pap, Triangular norms. Kluwer Acad. Publ., Dordrecht 2000.

[6] C. M. Ling, Representation of associative functions. Publ Math Debrecen, 12 (1965) 189-212.

[7] S. Saminger-Platz, R. Mesiar, D. Dubois, Aggregation operators and commuting, IEEE Transactions on Fuzzy Systems, 15 (2007) 1032-1045.

[8] H. Wang, F Qin, On the cross-migrativity of triangular subnorms, IEEE International Conference on Fuzzy Systems, (2014) 1139-1142. 